\title{
MODIS Aerosol Optical Thickness Product Algorithm Verification and Analysis
}

Aerosol and Air Quality Research

\author{
Yulong He, Lin Sun*, Zhendong Sun, Xueqian Hu \\ College of Geodesy and Geomatics, Shandong University of Science and Technology, Shandong, \\ Qingdao 266590, China
}

\section{ABSTRACT}

A Moderate Resolution Imaging Spectroradiometer (MODIS) aerosol optical thickness inversion algorithm has been rapidly developed in recent years, forming various representative aerosol optical thickness remote sensing products. Typical inversion algorithms include Dark Target, Dark Target-Deep Blue (DTB), and the high-spatial-resolution aerosol retrieval algorithm based on a priori land surface (HARLS). Both DTB and HARLS can realise aerosol optical depth inversion over land worldwide, but their accuracy and space-time adaptability vary. In this study, 80 Aerosol Robotic Network data worldwide were selected as the evaluation basis to quantitatively analyse and verify the adaptability and accuracy of different aerosol inversion algorithms on various underlying surfaces. The results indicate that the DTB algorithm has a higher inversion accuracy in vegetation area, whereas HARLS algorithm performs better in urban area; the HARLS and DTB algorithm accuracies in desert area type were found to be similar. The results provide a basis for selecting appropriate aerosol optical thickness remote sensing products for different applications.

Keywords: Aerosol optical depth, Prior database algorithm, Moderate Resolution Imaging Spectroradiometer, Adaptability analysis

\section{INTRODUCTION}

\section{OPEN ACCESS}

Received: February 4, 2021

Revised: August 26, 2021

Accepted: August 29, 2021

\section{* Corresponding Author: sunlin6@126.com}

\section{Publisher:}

Taiwan Association for Aerosol Research

ISSN: $1680-8584$ print

ISSN: 2071-1409 online

\section{(c) Copyright: The Author(s).}

This is an open access article distributed under the terms of the Creative Commons Attribution License (CC BY 4.0), which permits unrestricted use, distribution, and reproduction in any medium, provided the original author and source are cited.
Atmospheric aerosol is a mixture of minuscule solid and liquid particles that are suspended in the atmosphere. Aerosols substantially impact global and regional climate changes. Aerosols in the air can also affect human production, life, and health (Charlson et al., 1992; King et al., 1999). Precise atmospheric aerosol monitoring is significant to the study of climate change and environmental pollution. Conventional monitoring methods are primarily conducted using monitoring ground stations. However, because aerosol optical depth (AOD) changes rapidly over time and space, ground-based observations are limited by measuring equipment, site density, and observation range (Holben et al., 2001). It is difficult to achieve large-scale aerosol monitoring and perform macro-analysis and research on aerosol problems (Holben et al., 1996). Therefore, satellite remote sensing observation methods are widely used in the study of global and local aerosol spatial distribution and monitoring (Holben et al., 2001). Remote sensing predominately monitors the optical properties of aerosols. The AOD is only one of the important retrieval parameters, and it reflects the attenuation of solar radiation by particulate matter in the atmosphere.

In recent years, many researchers have conducted in-depth research on AOD inversion algorithms, proposing a variety of algorithms, including the Dark Target (DT) algorithm (Kaufman et al., 1997a), Deep Blue (DB) algorithm (Hsu et al., 2004), high-resolution aerosol retrieval algorithm with a priori land surface reflectance (LSR) database support (Wei and Sun, 2018) (HARLS), structural Function algorithm (Tanre et al., 1992), and polarisation algorithm (Cheng et al., 2015). Of these, the DT, DB, and HARLS algorithms are the most representative.

The DT algorithm was first proposed by Kaufman for vegetation areas. Kaufman found that in dense vegetation areas, the LSR of blue and red channels are low and exhibited a fixed relationship with the LSR of $2.12 \mu \mathrm{m}$. Later, researchers found that the linear relationship between the LSR in 
the 2.12- $\mu \mathrm{m}$ channel and red-blue channels was not stable but changed with the observation geometry. On this basis, the LSR in red-blue channels were expressed as a function of the scattering angle $(\theta)$ and vegetation index, which further improved the LSR accuracy in the red-blue channels and reduced the aerosol inversion error (Levy et al., 2007). The DT algorithm achieves superior inversion results in vegetation areas compared to the other algorithms, but it performs poorly in certain high LSR areas. To solve this problem, Hsu et al. (2004) proposed the DB algorithm for aerosol inversion in bright areas, which is based on the fact that the LSR in the blue band is low and stable. The DB algorithm uses Sea-viewing Wide Field-of-view Sensor (SeaWiFS) LSR data after atmospheric correction and minimum synthesis technology to construct a blue channel seasonal LSR database, and it uses multi-channel albedo and brightness temperature at $11 \mu \mathrm{m}$ and $12 \mu \mathrm{m}$ for cloud detection. Inverting aerosols using the DB algorithm can reduce the influence of observation geometry and Bidirectional Reflectance Distribution Function (BRDF) on the surface, and it can make up for the bright areas that the DT algorithm cannot invert (Wei et al., 2019). The latest Moderate Resolution Imaging Spectroradiometer (MODIS) products combine the advantages of DT and DB algorithms into a new algorithm (DTB). The DTB algorithm judges the surface type by setting the normalised difference vegetation index (NDVI) threshold, and it chooses the corresponding inversion algorithm according to the underlying surface type. Therefore, the DTB algorithm can achieve highly accurate optical depth inversion for different surface types.

HARLS, proposed by Sun et al. (2018), realised the global high-resolution inversion of terrestrial AOD by constructing a priori global LSR and aerosol type databases (Wei, 2017). Unlike the DB algorithm, HARLS uses MOD09A1 LSR data to construct a blue channel LSR database and uses the dynamic threshold method to detect clouds. Using MOD04 aerosol products and by synthesising seasonal aerosol type databases, the HARLS algorithm can accurately retrieve aerosols in bright areas with a high spatial resolution $(1 \mathrm{~km})$. Both the DTB and HARLS algorithms can realise AOD inversion over land worldwide. Therefore, it is necessary to validate the accuracy and adaptability of the two algorithms for AOD inversion.

In recent years, the quality of AOD products has been a widely discussed issue in the scientific community. Many scholars have conducted in-depth research on the performances and adaptabilities of various aerosol products (Yang et al., 2016). Li et al. (2012) verified the accuracy of MODIS aerosol products in Northwest China using the AOD values observed by solar photometers at four locations in Xinjiang. The results demonstrated that both MODIS C4 and C5 products overestimated AOD values. They noted that both the aerosol model and LSR estimation were the reasons for this overestimation. Sayer et al. (2013) used data from 60 Aerosol Robotic Network (AERONET) sites to study the accuracy and uncertainty of C6 DB products. Wang et al. (2017) validated the global accuracy of the MODIS DT and DB algorithms and evaluated the impact of surface reflectance and aerosol models on algorithm accuracy. Aerosol product adaptability analyses can be divided into regional and global validations. At present, most studies have focused on the validation and analysis of aerosol products in a specific region, and there is a lack of in-depth analysis of the adaptability of the DTB and HARLS algorithms both for different regions and globally.

This study aims to evaluate the AOD inversion accuracy of the DTB and HARLS algorithms. Longterm sequence data of MOD04 C6.1 DTB (10 km) and HARLS AOD (1 km) from 2014 to 2016 were obtained. AERONET ground-based observation data, from typical underlying surfaces with large difference in surface types, were selected to verify and compare the aerosol products. The adaptabilities of these two different aerosol inversion algorithms on a global scale and in different regions were assessed, providing a basis for selecting appropriate AOD remote sensing products for different applications and improving the accuracy of the inversion algorithm (Zhou et al., 2009).

\section{METHODS}

\subsection{Inversion Algorithms}

\subsubsection{HARLS}

The apparent reflectance observed by satellite sensors comprises LSR and atmospheric contributions, as illustrated in Eq. (1). 
$\rho_{T O A(\theta s, \theta v, \psi)}=\rho_{\operatorname{Aer}(\theta s, \theta v, \psi)}+\rho_{\operatorname{Ray}(\theta s, \theta v, \psi)}+\frac{\rho_{\text {Sur }}}{1-\rho_{\text {Sur }} \cdot S} T(\theta s) T(\theta v)$

The LSR $\rho$ sur is unknown. Certain parameters can be written as a function of optical thickness $\tau$. The aerosol model provides two parameters: single scattering albedo and asymmetric factor. Thus, the aerosol inversion algorithm faces two primary problems: LSR estimation and aerosol type selection (Sun et al., 2016). The complexity and diversity of land surface structures make it difficult to estimate the LSR. The diversity of aerosol types changes over time and space, making it difficult to invert the AOD. HARLS constructs a blue-channel LSR database using MODIS 8-d synthetic LSR products. To eliminate the influence of clouds and atmospheric correction error, a monthly surface reflectance database was constructed using the minimum synthesis method, which provides more accurate surface reflectance for aerosol inversion. The database used is the synthesis of surface reflectance products from 2010 to 2014, which is different from the current year land surface reflectance database used by Wei et al. (2018). For cloud detection, the reflectance of the underlying surface was provided according to the established LSR database; then, the $6 \mathrm{~S}$ radiation transfer model was used to simulate the change in the apparent reflectance under different observation and atmosphere conditions. If the real apparent reflectance of a pixel, that is, the apparent reflectance provided in the image is greater than the change of the simulated apparent reflectance maximum value, then the pixel is considered a cloud pollution pixel (Wei, 2017). For aerosol type selection, Levy et al. (2013) used the aerosol robotic network (AERONET) to measure aerosol optical properties and conducted a new cluster analysis on aerosol types; the results showed that the measured values of most AERONET sites remained unchanged in the global pat tern. Therefore, we assume that the dominant aerosol types at each observation site are a function of the season (Levy et al., 2007; Sun et al., 2016b). We then use the daily aerosol type data provided by MODIS aerosol products to obtain all MOD04 aerosol type data in a quarter; the mode of all data aerosol type values was taken as the aerosol type of the pixel, so as to establish the global aerosol type dataset in different seasons (Fig. 1). The spatial resolution of the global aerosol type database is $1 \mathrm{~km}$, and the values 1 to 5 represent the following: 1: continental aerosol, 2: moderate absorption model, 3: strong absorption model, 4: weak absorption model, and 5: dust aerosol. MOD02 1-km data were used as input data; therefore, HARLS can be used to retrieve the AOD over land at a high resolution $(1 \mathrm{~km})$.

\subsection{MODIS Aerosol Products}

MODIS datasets provide two aerosol products with long time series and global coverage: second-level daily aerosol products with resolutions of $10 \mathrm{~km}$ (MxD04_L2, where O represents the Terra satellite and $Y$ represents the Aqua satellite) and $3 \mathrm{~km}$ (MxD04_3K). This study verifies the accuracy of the DTB algorithm and HARLS; therefore, the MOD04_L2 (DTB) dataset was selected. To ensure the objectivity of the comparison results, HARLS utilised the same data source as that of the DTB algorithm (MOD02 $1 \mathrm{~km}$ ).

\subsubsection{MODIS DTB algorithm}

The DT algorithm exhibits sufficient inversion accuracy in dense vegetation areas. The LSR of vegetation varies greatly over time; thus, it is particularly important to determine the real-time LSR of vegetation for AOD inversion accuracy. Based on Kaufman (Kaufman et al., 1997b; Kaufman and Sendar, 1988; Gupta, 2016), Levy et al. (2007) found that the linear relationship between $2.12 \mu \mathrm{m}$ and red-blue channels LSR is not fixed but changes with the observed geometric conditions. Therefore, a more accurate method for estimating LSR is proposed. The red and blue channel LSR is expressed as a function of scattering angle $(\theta)$ and vegetation index:

$\rho 0.66=f(\rho 2.12)=\rho 2.12 \times$ slope $0.66 / 2.12+y$ int $0.66 / 2.12$

$\rho 0.47=g(\rho 0.66)=\rho 0.66 \times$ slope $0.47 / 0.66+y$ int $0.47 / 0.66$

where $\rho 2.12, \rho 0.66$, and $\rho 0.47$ represent the LSR at $2.12-\mu \mathrm{m}, 0.66-\mu \mathrm{m}$, and $0.47-\mu \mathrm{m}$ channels. Therefore, the DT algorithm can estimate the LSR of vegetation area more accurately and retrieve AOD with higher accuracy (Sun et al., 2016). 


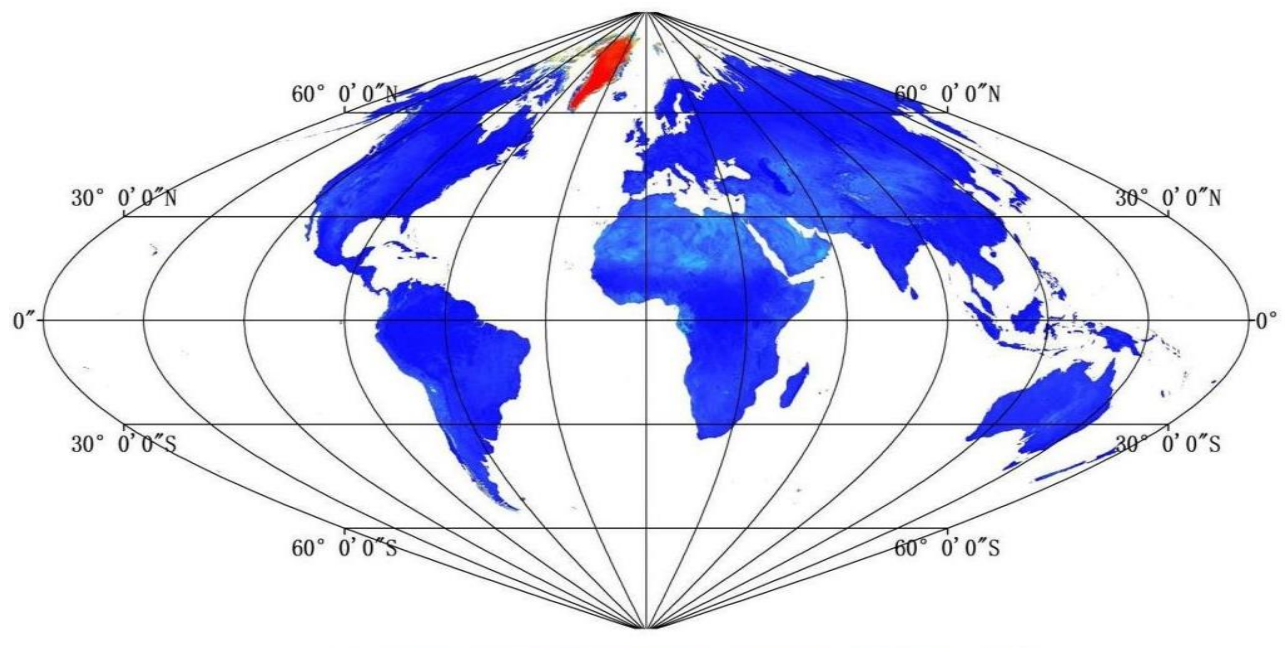

0

(a)



(b)

Fig. 1. (a) Blue band image of global 1-km surface reflectance and (b) distribution map of global aerosol types.

The DB algorithm can effectively retrieve AOD in high reflectivity regions. Certain highlighted areas, such as cities and deserts, are less affected by the phenological period and their LSRs do not change significantly over time. Therefore, the DB algorithm uses minimum synthesis technology to construct a blue channel LSR database for multi-channel SeaWiFS surface reflectance data to provide the LSR. The latest DB algorithm also improves the surface reflectance estimation. Using an LSR database and the NDVI, a hybrid method was used to determine the LSR under different land cover types.

Therefore, the DT and DB algorithms exhibit different advantages for various underlying surface types. The DTB algorithm combines these two algorithms and selects the appropriate algorithm according to the NDVI threshold setting: (1) If NDVI > 0.3, use the DT algorithm: (2) if $\mathrm{NDVI}<0.2$, use the DB algorithm; (3) if $0.2 \leq \mathrm{NDVI} \leq 0.3$, take the average value of the inversion results of the DT and $\mathrm{DB}$ algorithms or select a data set with high-quality control (QA = 3 for DT algorithm and QA = 2 for DB algorithm). Therefore, the DTB algorithm can achieve AOD inversion over land with high accuracy. However, due to the limited resolution of the SeaWiFS data, the resolution of the AOD products produced by the DTB algorithm $(10 \mathrm{~km})$ is lower than that of HARLS $(1 \mathrm{~km})$. 


\subsection{AERONET Data}

AERONET is a globally calibrated terrestrial aerosol observation network that uses CE-318 solar photometer measurements to collect data. AERONET can provide AOD at $380 \mathrm{~nm}, 440 \mathrm{~nm}, 500 \mathrm{~nm}$, $675 \mathrm{~nm}, 870 \mathrm{~nm}$, and $1020 \mathrm{~nm}$, with observation errors ranging from 0.01 to 0.02 (Holben et al., 1998), and three levels of AOD measurements: L1.0 (unscreened), L1.5 (cloud screening), L2.0 (cloud screening and quality assurance). L2.0 data exhibits higher quality control and reliability; hence, we selected $\mathrm{L} 2.0$ as the validation data.

The central wavelength and spatial-temporal scale corresponding to the MODIS data and AERONET site data, respectively, do not match; therefore, data interpolation and spatiotemporal matching were required to realise the effective verification comparison of site data and aerosol products. Using AERONET data available at $500 \mathrm{~nm}$ and $675 \mathrm{~nm}$ for AOD measurements, AOD values at $550 \mathrm{~nm}$ were interpolated using the Ångström algorithm (1964) (Eqs. (3)-(5)).

$A O D \lambda i=\beta \lambda i^{-\alpha}$

$\alpha=\frac{\ln (A O D \lambda 1 / A O D \lambda 2)}{\ln (\lambda 1 / \lambda 2)}$

$\beta=\frac{A O D \lambda 1}{\lambda i^{-\alpha}}$

where $A O D \lambda i$ is the aerosol optical thickness of wavelength $\lambda i, \beta$ is the turbidity coefficient of Ångström, and $\alpha$ is the wavelength index, which is related to the aerosol average radius. $\lambda 1, \lambda 2$ represent the wavelengths at $500 \mathrm{~nm}$ and $675 \mathrm{~nm}$, respectively. To improve the reliability of the data comparison, the AERONET site data and aerosol products were matched in both time and space. Time matching was based on the transit time of the satellite, and the AOD value of the station was selected and averaged within $30 \mathrm{~min}$ before and after that time. Spatial matching was based on the spatial average of aerosol data in $5 \times 5$ pixels centred on the station.

\subsection{Evaluation Methods}

The Pearson correlation coefficient method was used to calculate the correlation coefficient ( $r$ ) between the inverted AOD values and the AERONET ground-based AOD values. The expected error (EE), mean absolute error (MAE), root mean square error (RMSE), and relative mean bias (RMB) (Bilal et al., 2016; Georgoulias and Zanis, 2016; Almazroui, 2019) were calculated to quantitatively evaluate the uncertainty of aerosol inversion. A RMB greater than 1 or less than 1 indicates that the AOD values of aerosol inversion are overestimated or underestimated, respectively.

$$
r=\frac{\sum_{i=1}^{n}(x i-\bar{x})(y i-\bar{y})}{\sqrt{\sum_{i=1}^{n}(x i-\bar{x})^{2} \sum_{i=1}^{n}(y i-\bar{y})^{2}}}
$$

$E E= \pm\left(0.05+0.15 \times A O D_{A E R O N E T}\right)$

$M A E=\frac{1}{n} \sum_{i=1}^{n}\left|\left(A O D_{\text {satellite }}-A O D_{\text {AERONET }}\right)\right|$

$R M A E=\sqrt{\frac{1}{n} \sum_{i=1}^{n}\left(A O D_{\text {satellite }}-A O D_{\text {AERONET }}\right)^{2}}$

$R M B=\frac{1}{n} \sum_{i=1}^{n}\left|\frac{A O D_{\text {Satellite }}}{A O D_{\text {AERONET }}}\right|$ 


\section{RESULTS AND DISCUSSION}

\subsection{HARLS and DTB Algorithms Global-scale Verification}

In the study, we selected 80 global AERONET sites to verify the aerosol product accuracies. Fig. 2 compares the HARLS and DTB algorithms with AERONET station observations. The data points exhibit the expected error. The HARLS and DTB algorithms inversion results agree with those of the AERONET site data $\left(R_{H}=0.8220\right.$ and $\left.R_{D T B}=0.8720\right)$. The RMB of both algorithms is greater than 1 , which indicates that they overestimate the AOD value. This is because the HARLS and DTB algorithms adopt the minimum synthesis method to build an LSR database. Under atmospheric pollution conditions, the underestimation of LSR produces high AOD values. Moreover, both algorithms exhibit similar MAEs $\left(\mathrm{MAE}_{\mathrm{H}}=0.0988\right.$ and $\left.\mathrm{MAE} \mathrm{E}_{\mathrm{DTB}}=0.0822\right)$ and $\mathrm{RMSEs}\left(\mathrm{RMSE}_{\mathrm{H}}=0.15\right.$ and RMSEDTB $=0.13)$; however, the DTB algorithm exhibits a higher inversion rate $(69.95 \%)$ within the expected error than the HARLS algorithm (61.02\%). As displayed in Fig. 3, when the AOD value is small (AOD $<0.5$ ), more DTB algorithm points fall within the expected error line, while when the AOD value is large, (AOD > 0.5) HARLS exhibits a higher success rate. To illustrate this phenomenon in greater detail, we classify AOD values into different levels (A: 0-0.1, B: 0.1-0.2, C: $0.2-0.5, D: 0.5-1.0$, and $E:>1.0$ ) and calculate the MAE for each level. As illustrated in Fig. 4, when the AOD value is less than 0.2, the DTB algorithm MAE is lower than that of HARLS. However, with an increase in the AOD value (AOD > 0.5), HARLS exhibits a higher inversion accuracy. In vegetation areas, AOD values are typically smaller owing to less human activity, while those of urban areas are subject to greater human activity and industrial emissions and thus exhibit higher AOD values. This demonstrates that the two algorithms exhibit various adaptabilities under different surface types. To understand the differences between the two algorithms, their accuracies and adaptabilities under different surface types were analysed in depth.

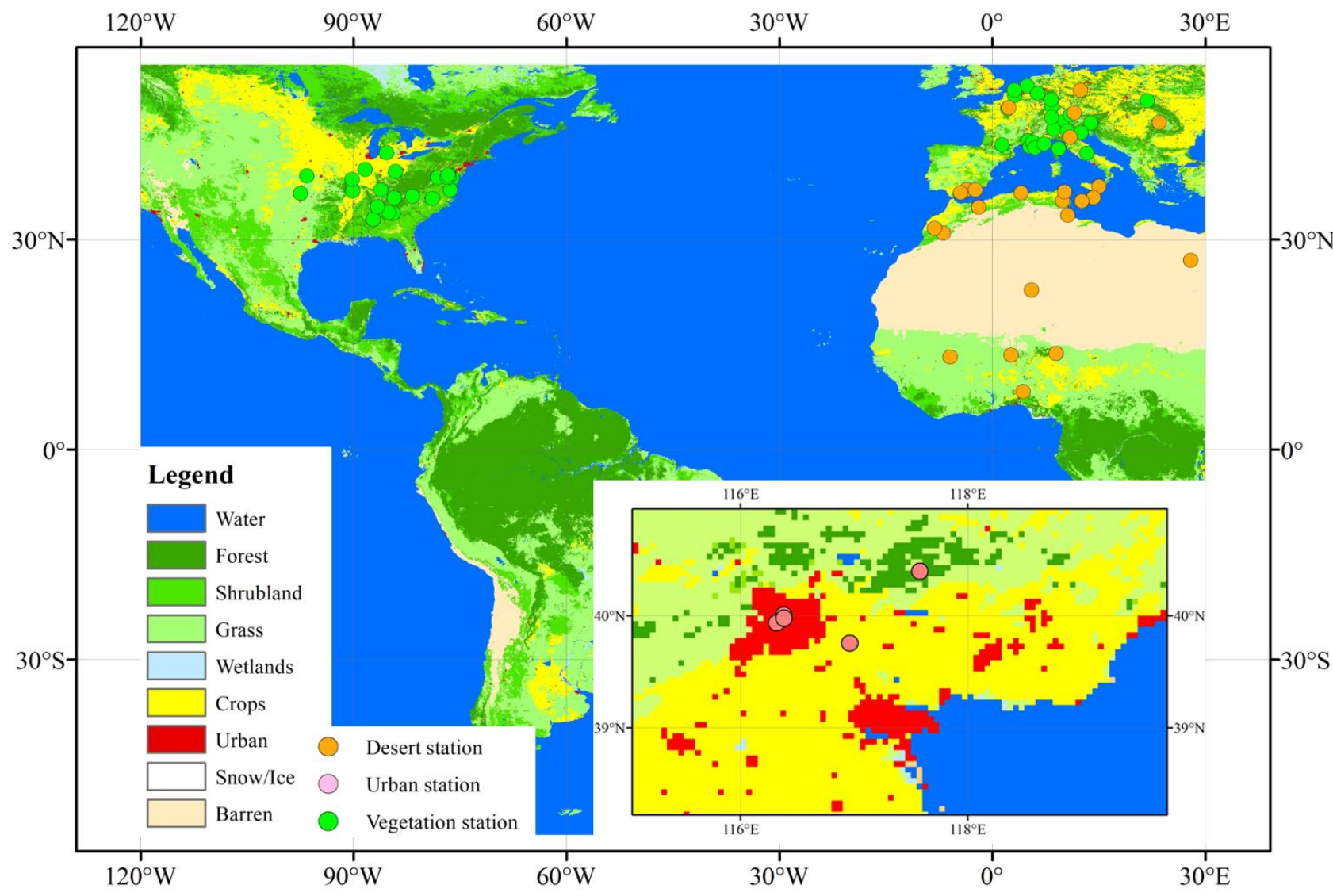

Fig. 2. Distribution map of part AERONET sites. (The base map uses MODIS land classification products, the small map shows the distribution of sites in the urban area). 




(a)

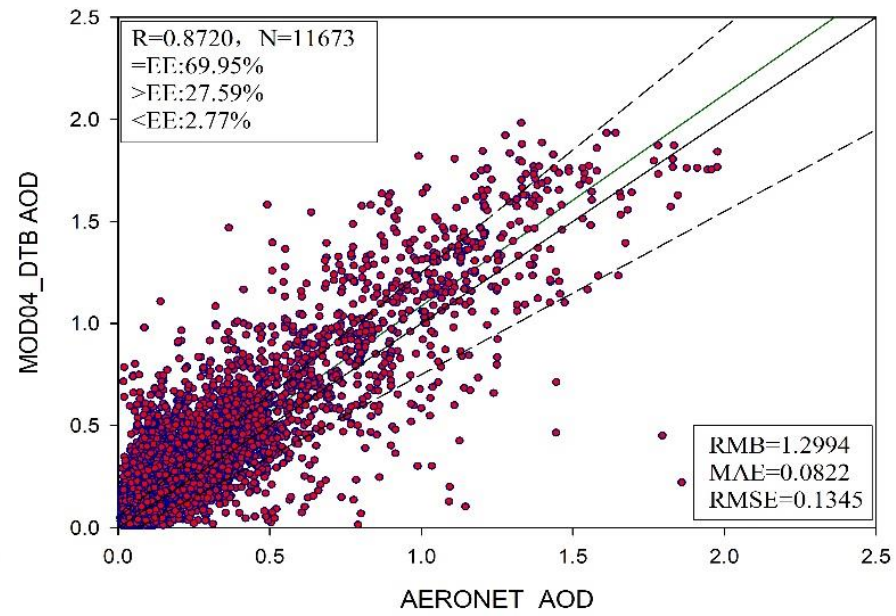

(b)

Fig. 3. (a) HARLS and (b) MOD04_DTB AOD validations. Green solid line represents data point line of best fit, black line represents $\mathrm{Y}=\mathrm{X}$, and dotted lines represent error lines.

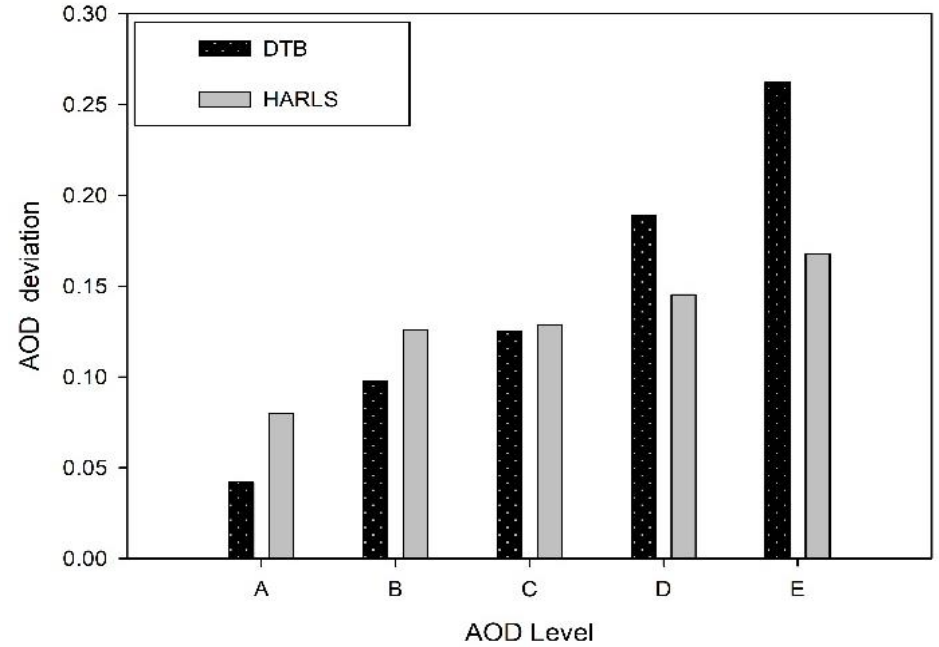

Fig. 4. Deviation histograms for different AOD ranges.

\subsection{HARLS and DTB Algorithm Regional Adaptability Analysis}

Owing to changes in geography, climate, and human activity, AOD inversion algorithms exhibit different adaptabilities at various regional levels. The reflectance of the vegetation area is affected by the vegetation growth rule. The complex surface type and BRDF of urban areas greatly impact their LSR. The sand and dust weather in arid and semi-arid areas also make LSR determination more difficult. There are significant differences between the HARLS and DTB algorithms in determining LSR, leading to differences in algorithm adaptability across regions (Yang et al., 2016). In this study, the HARLS and DTB algorithms adaptability under different land surface types is tested and compared for middle eastern North America (MENA), Middle Europe (ME), arid Northwest Africa (NA), and Beijing-Tianjin-Hebei (BTH).

\subsubsection{Vegetation area}

MENA and ME are typical vegetation cover areas. The climate is primarily temperate continental that is suitable for vegetation growth, particularly sub-frigid coniferous forest. The region is characterised by high vegetation coverage, accounting for approximately $30 \%$ of the global total (Wei et al., 2018). Early industrial development polluted the air in this area, primarily with fine particle and aerosol-type pollutants with weak absorption (Wei, 2017). Thirty-one AERONET sites 


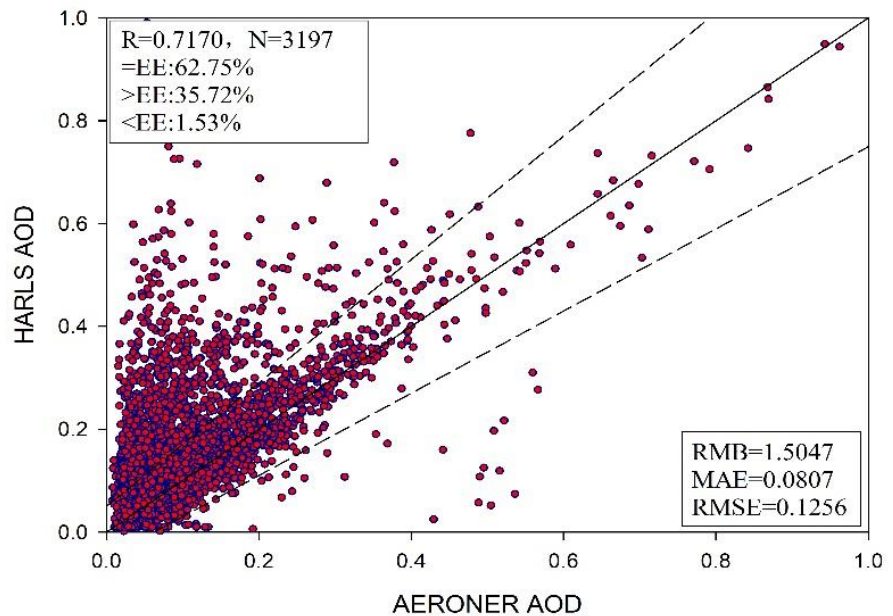

(a)

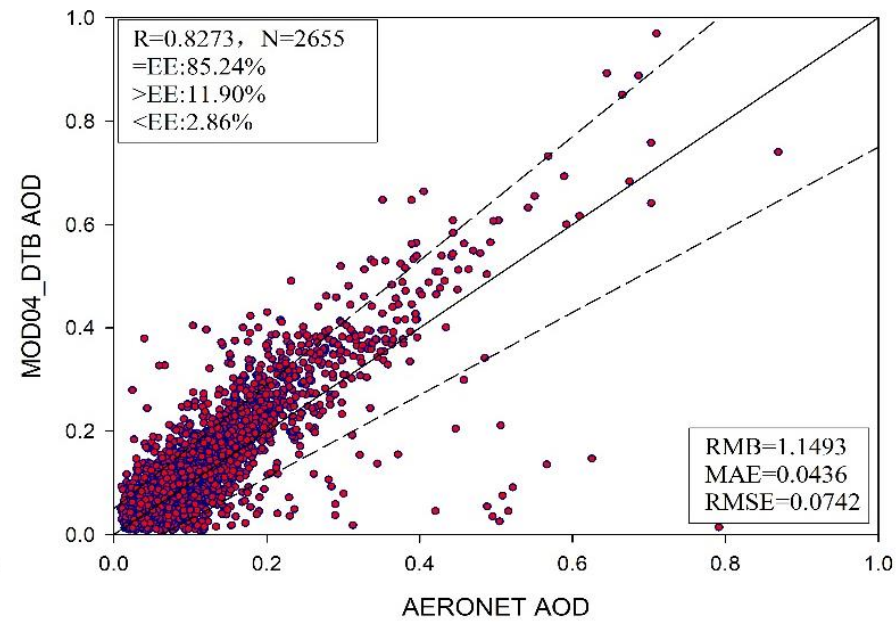

(b)

Fig. 5. (a) HARLS and (b) MOD04_DTB AOD validations in vegetation area. Black line represents $Y=X$, and dotted lines represent error lines.

were selected in this area to verify the two algorithms (Fig. 5 is a comparison of HARLS and the DTB algorithm for AOD inversion in MENA). As illustrated in Fig. 5, the DTB algorithm is superior to HARLS for all accuracy indicators, and it exhibits greater consistency with AERONET site data. For the DTB algorithm, $85 \%$ of the points fall within the expected error line, which is higher than that for HARLS (63\%). The MAE (0.04) and RMSE (0.07) for the DTB algorithm were also smaller than those of HARLS (MAE $=0.08$ and RMSE $=0.12$ ). This is primarily because MENA is a high vegetation-coverage area. The DTB algorithm selects the DT algorithm to invert the AOD value in this area. The DT algorithm determines the LSR based on the close relationship between the 2.12- $\mu \mathrm{m}$ and red-blue channels, which is more stable in the vegetation area. Therefore, the DT algorithm can accurately determine the vegetation LSR to invert the AOD value with high accuracy. In contrast, vegetation LSR varies greatly in the growing season. HARLS establishes an LSR database using the minimum synthesis method. Therefore, in the vegetation growing season, HARLS underestimates the vegetation LSR, resulting in an overestimation of the AOD value, which also explains why the HARLS RMB (1.5047) is higher than that of the DTB RMB (1.1493). Moreover, the vegetation area AOD values are small, with $95 \%$ of the values being less than 0.5 (Fig. 5); this further confirms that the DTB algorithm exhibits a high inversion accuracy in vegetation areas where the aerosol type is primarily weak absorption.

\subsubsection{Urban areas}

BTH is the largest and most productive urban area in northern China. BTH exhibits a complex surface structure, including several bright surfaces and urban areas with artificial buildings and sparse vegetation (Wang et al., 2012). The regional aerosol models primarily exhibit weak and medium absorption (Wei et al., 2018). As displayed in Fig. 6, the HARLS and DTB algorithms inversion accuracy in urban areas is lower than that in vegetation areas. First, the urban area surface types are complex, and the relationship between the red and blue channels of these land surface types with the LSR of the $2.1-\mu \mathrm{m}$ channel exhibits more complex behaviour that is inconsistent with that of natural vegetation areas. Therefore, when DTB selects the DT or DB algorithm for AOD inversion, it results in poor inversion accuracy. Second, the LSR of urban areas is high, and it is also vulnerable to seasonal variations of vegetation growth and death stages, and BRDF. Therefore, both the HARLS and DTB algorithms produce errors in their LSR estimations for urban areas. However, compared with the HARLS and DTB algorithm accuracies in urban areas, the HARLS inversion results are more consistent with AERONET site data, and more points satisfy the expected errors as exhibited by the smaller RMB and MAE. Generally speaking, HARLS exhibits higher inversion accuracy than the DTB algorithm in urban areas. This is primarily because urban surface heterogeneity is a problem in AOD inversion for urban areas. For example, when vegetation is near a building, the LSR of the entire pixel changes. Improving pixel resolution 


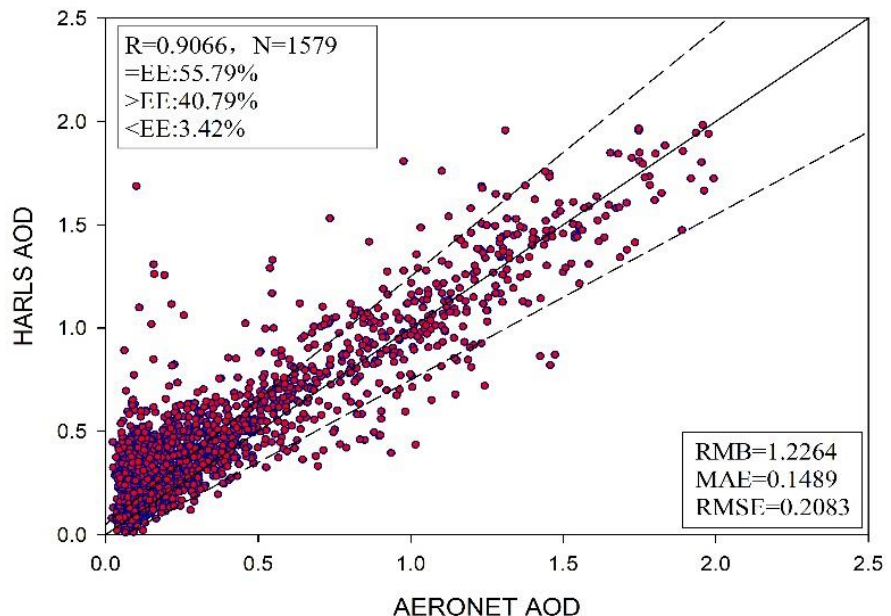

(a)

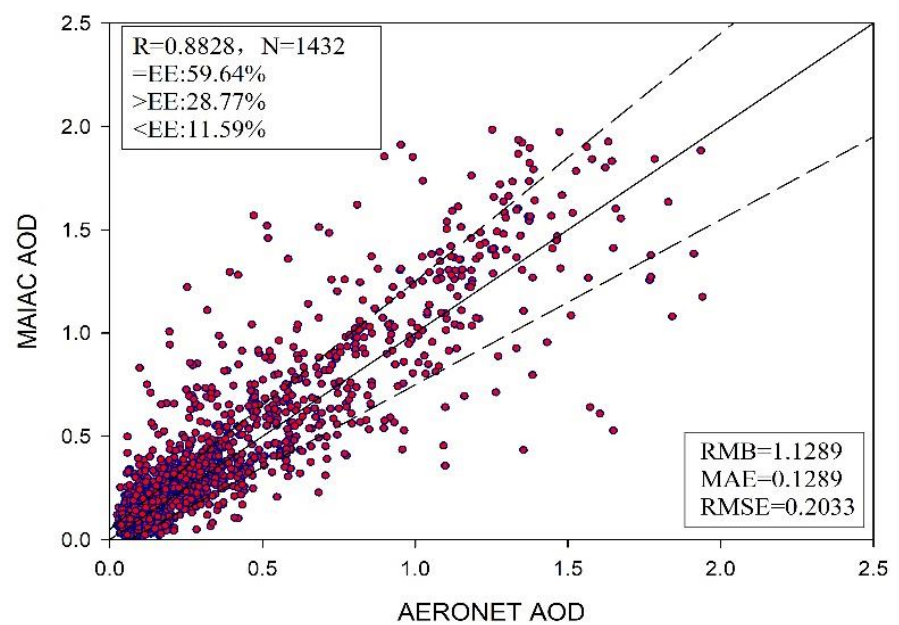

(c)

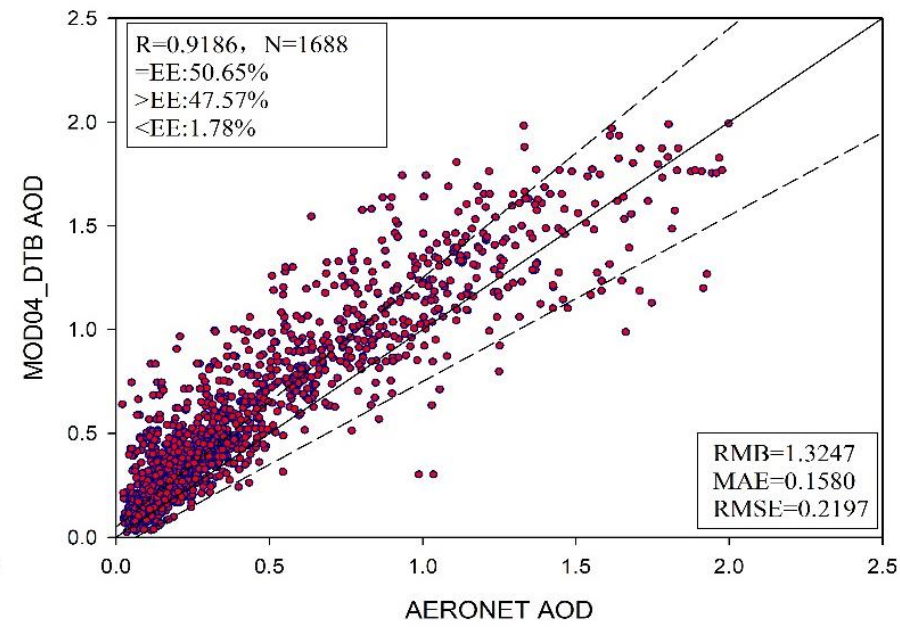

(b)

Fig. 6. (a) HARLS, (b) MOD04_DTB, and (c)MAIAC AOD validations in urban area. Black line represents $Y=X$, and dotted lines represent error lines.

can effectively reduce the impact of surface heterogeneity on estimating LSR. The DTB algorithm exhibits a resolution of $10 \mathrm{~km}$ due to the limited SeaWiFS database resolution, while HARLS uses MOD09A1 data to establish a 1-km resolution LSR database. To further verify the influence of resolution on the inversion accuracy of urban area, Multi-Angle Implementation of Atmospheric Correction (MAIAC, 1-km resolution) aerosol products are included and compared with those of the other algorithms. The MAIAC algorithm uses a time series method to obtain the surface reflection relationship between MODIS blue and shortwave infrared bands in dark and bright regions and considers the influence of bidirectional surface reflection. The MAIAC algorithm not only improves the spatial resolution of the product but also the inversion accuracy in bright areas. Wang (2021) verified the adaptability of MODIS MAIAC AOD products in China from 2008 to 2016; the MAIAC inversion results performed well in Beijing urban area ( $E E=57.12 \%, \mathrm{RMSE}=0.20$ ). Compared with the DTB algorithm, HARLS and MAIAC have more points in the expected error line and a higher accuracy; the verification accuracy is consistent with Wang's verification results in urban areas. It has also been verified that the higher spatial resolution can improve the inversion accuracy in urban areas (Wang et al., 2021). Therefore, the HARLS and MAIAC algorithms can achieve a higher inversion accuracy in urban areas than the DTB algorithm.

\subsubsection{Desert region}

NA primarily includes the Sahara Desert. The regional climate is predominately arid and tropical, with high temperatures and low vegetation coverage. Owing to the influence of the Sahara Desert, 


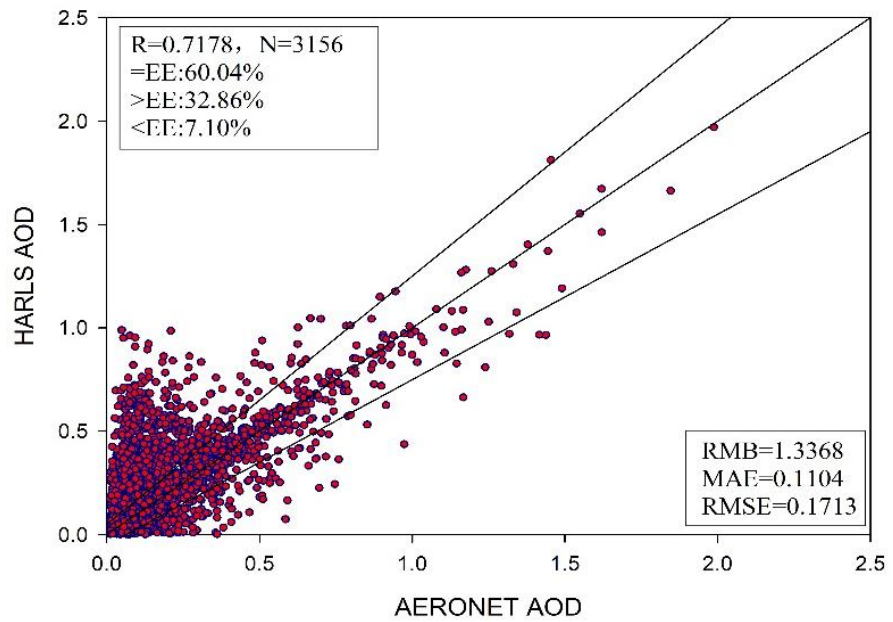

(a)

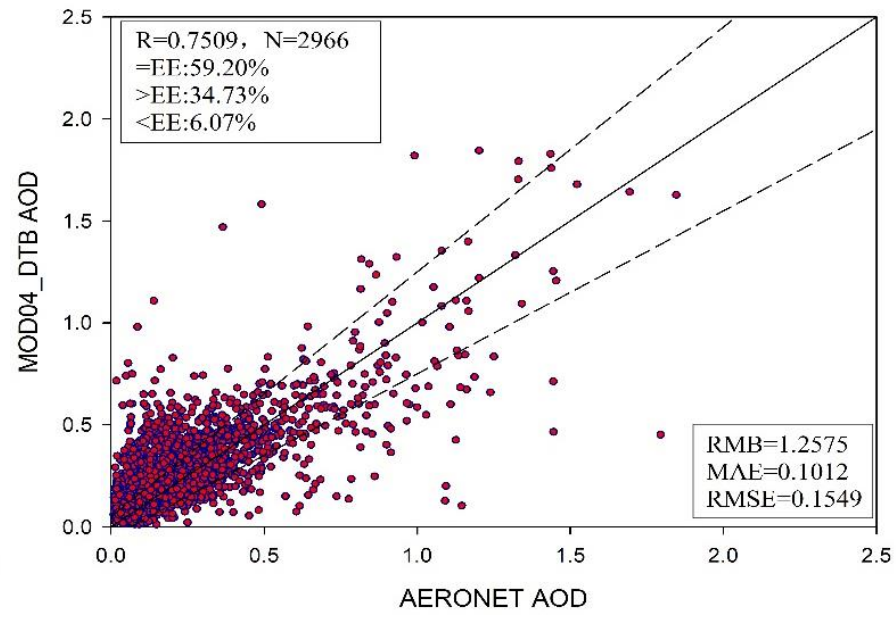

(b)

Fig. 7. (a) HARLS and (b) MOD04_DTB AOD validations in desert area. Black line represents $Y=X$, and dotted lines represent error lines.

the region has become a primary source of dust weather (Hsu et al., 2004). The overall regional reflectance is higher than that of other regions because of the frequent dust weather and lack of vegetation coverage. Twenty-six AERONET sites were selected in this area for comparative verification. Fig. 7 displays a comparison and validation chart of HARLS and the DTB algorithm for aerosol inversion in desert areas. As illustrated in the figure, the consistency between AOD inversions by HARLS and the DTB algorithm with that of the AERONET site data is lower than that of vegetation and urban areas. The HARLS and DTB algorithms perform poorly in desert areas. First, the overall desert area reflectance is higher than that of other area types. With the increase in reflectance, the aerosol optical thickness sensitivity to apparent reflectance decreases. Second, frequent dust activity and complex surface information in desert areas make it difficult to determine the LSR. The HARLS and DTB algorithm accuracies in this area type are similar. The Northern Africa area is primarily desert; thus, the influence of surface heterogeneity can be neglected. Therefore, the high-resolution characteristics of HARLS do not exhibit and advantage in desert areas.

\subsection{HARLS and DTB Algorithm Spatial Comparative Analysis}

Fig. 8 shows the spatial distribution of HARLS and DTB algorithms for different underlying surfaces. The results indicate that the aerosol distribution of HARLS algorithm in three typical areas (urban, vegetation, and desert areas) shown in (a), (c) and (e); (b), (d) and (f) show the spatial distribution of DTB in different regions. DTB and HARLS algorithm exhibit better spatial continuity under different underlying surfaces. The primary reason is that the HARLS algorithm can realize different underlay AOD inversion by building global LSR database, the DTB algorithm employs the DT algorithm in dense vegetation area for inversion; however, the DT algorithm cannot achieve inversion for high-density surface underlay-type, in which case, the DB algorithm is used for AOD inversion. Therefore, both algorithms can realize AOD inversion under different surface types on a global scale. However, the overall inversion of AOD value of DTB algorithm in urban area is higher than that of HARLS algorithm. For vegetation area, the overall inversion of AOD value of HARLS algorithm is higher than that of DTB algorithm. This further verifies the regional verification results. Moreover, the HARLS algorithm has higher RMB value in vegetation area, while DTB algorithm has higher RMB value in urban area.

\section{CONCLUSIONS}

Based on AERONET ground observation data in the global and different land surface types, this study compares and analyses the adaptability of aerosol results retrieved using different algorithms. Based on the results, the following conclusions can be drawn. 


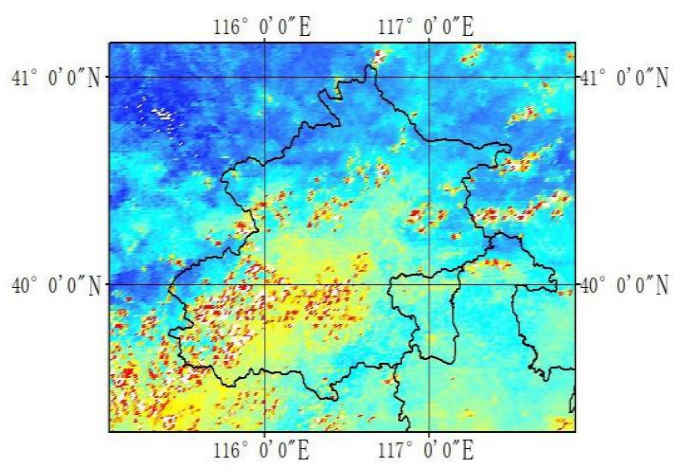

(a)

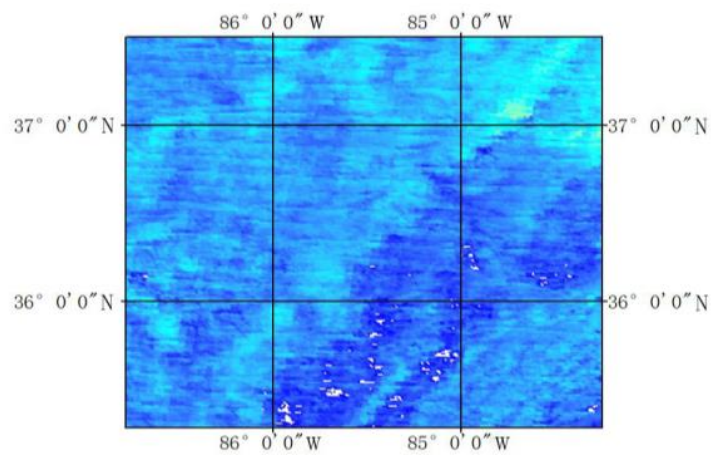

(c)

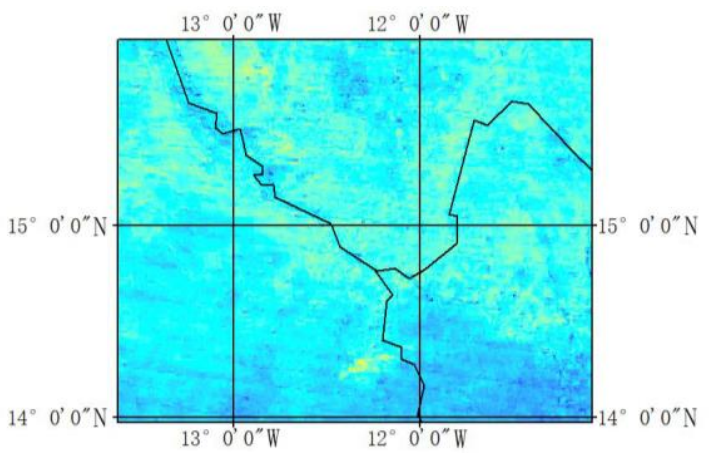

(e)

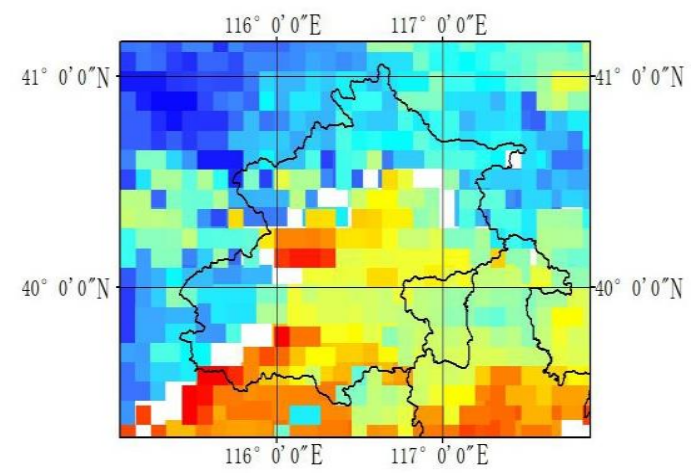

(b)

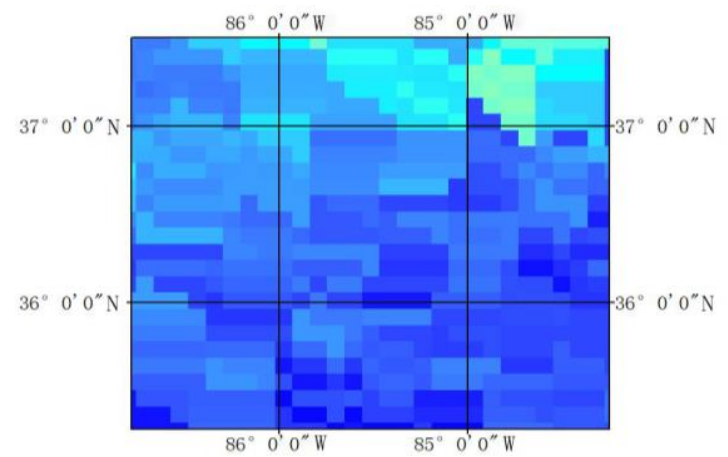

(d)

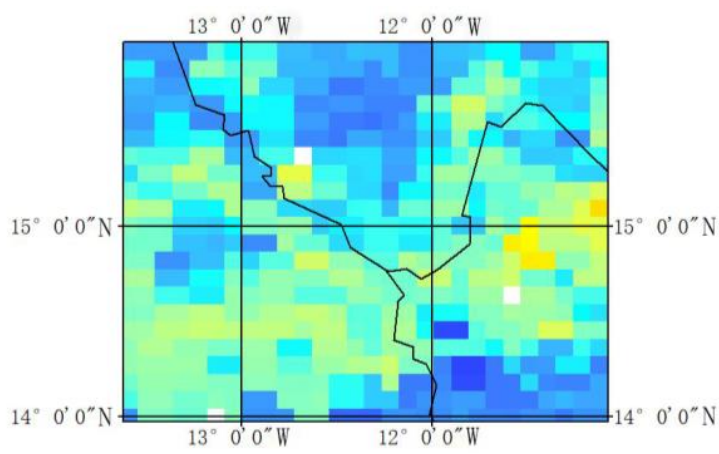

(f)

0

2.0

Fig. 8. Spatial contrast distribution of different aerosol products. (a) and (b), (c) and (d), and (e) and (f) show the spatial distributions of HARLS and DTB aerosol products in urban, vegetation, and desert areas, respectively.

1. Global-scale verification demonstrates that the DTB algorithm exhibits higher accuracy in the low AOD range, while HARLS exhibits superior performance in the high AOD range. This is because the DTB algorithm selects different algorithms for various surface types.

2. The DT algorithm can accurately estimate the LSR in vegetation areas in real-time, while HARLS is limited by a historical LSR database. Therefore, the DTB algorithm exhibits sufficient inversion accuracy in vegetation areas. HARLS exhibits superior performance to that of the DTB algorithm in urban areas because of the uneven urban surface. The high-resolution inversion characteristics of HARLS can be advantageous. Due to the bright and uniform surface of the desert area, HARLS and the DTB algorithm exhibit similar accuracy.

3. The methods employed to construct the database and choice of algorithm, allow the HARLS and DTB algorithms to realize AOD inversion for different land surface types worldwide; consequently, they exhibit a high spatial continuity.

4. The DTB algorithm combines the advantages of the DT and DB algorithms; however, its global verification results are suboptimal. This result indicates that it is not always correct to rely 
solely on a fixed NDVI threshold merging procedure, and various factors, such as land-use type, aerosol type, and pixel resolution (Wei et al., 2019), must also be considered.

\section{ACKNOWLEDGMENTS}

The authors thank NASA for providing the MODIS data (http://ladsweb.nascom.nasa.gov), and AERONET measurements are available from the NASA Godard Space Flight Center (https://aerone t.gsfc.nasa.gov/). This work was supported by the National Natural Science Foundation of China (No. 41771408) and Natural Science Foundation of Shandong province (No. ZR201702210379).

\section{DISCLAIMER}

The authors declare no conflict of interest.

\section{REFERENCES}

Almazroui, M. (2019). A comparison study between AOD data from MODIS deep blue collections 51 and 06 and from AERONET over Saudi Arabia. Atmos. Res. J. 225, 88-95. https://doi.org/10. 1016/j.atmosres.2019.03.040

Ångström, A. (1964). The parameters of atmospheric turbidity. Tellus 16, 64-75. https://doi.org/ 10.1111/j.2153-3490. 1964. tb00144.x

Bilal, M., Nichol, J.E., Nazeer, M. (2016). Validation of Aqua-MODIS C051 and C006 operational aerosol products using AERONET measurements Over Pakistan. IEEE J. Sel. Top. Appl. Earth Obs. Remote Sens. 9, 2074-2080. https://doi.org/10.1109/JSTARS.2015.2481460

Charlson, R.J., Schwartz, S.E., Hales, J.M., Cess, R.D., Coakley, J.J., Hansen, J.E., Hofmann, D.J. (1992). Climate forcing by anthropogenic aerosols. Science 255, 423-430. https://doi.org/10.1 126/science.255.5043.423

Cheng, C., Li, Z.Q., Hou, W.Z. (2015). Parasol multi angle polarization satellite aerosol optical depth retrieval algorithm based on dynamic aerosol model. J. Remote Sens. 19, 25-33. https://doi.org/10.11834/jrs.20154043

Georgoulias, A.K., Alexandri, G., Kourtidis, K.A., Lelieveld, J., Zanis, P., Amiridis, V. (2016). Differences between the MODIS Collection 6 and 5.1 aerosol datasets over the greater Mediterranean region. Atmos. Environ. 147, 310-319. https://doi.org/10.1016/j.atmosenv.2016.10.014

Gupta, P., Levy, R.C., Mattoo, S., Remer, L.A., Munchak, L.A. (2016). A surface reflectance scheme for retrieving aerosol optical depth over urban surfaces in MODIS dark target retrieval algorithm. Atmos. Meas. Tech. 9, 3293-3308. https://doi.org/10.5194/amt-9-3293-2016

Holben, B.N., Setzer, A., Eck, T.F., Pereira, A., Slutsker, I. (1996). Effect of dry season biomass burning on Amazon Basin aerosol concentrations and optical properties, 1992-94. J. Geophys. Res. 101, 19465-19481. https://doi.org/10.1029/96JD01114

Holben, B.N., Eck, T.F., Slutsker, I.A., Tanre, D., Buis, J.P., Setzer, A., Vermote, E., Reagan, J.A., Kaufman, Y.J., Nakajima, T., Lavenu, F. (1998). AERONET-A federated instrument network and data archive for aerosol characterization. Remote Sens. Environ. 66, 1-16. https://doi.org/10.1 016/S0034-4257(98)00031-5

Holben, B.N., Tanre, D., Smirnov, A., Eck, T.F., Slutsker, I., Abuhassan, N., Newcomb, W.W., Schafer, J.S., Chatenet, B., Lavenu, F.J.J.O.G.R.A., Kaufman, Y.J. (2001). An emerging groundbased aerosol climatology: Aerosol optical depth from AERONET. J. Geophys. Res. 106, 1206712097. https://doi.org/10.1029/2001JD900014

Hsu, N.C., Tsay, S.C., King, M.D., Herman, J.R. (2004). Aerosol properties over bright-reflecting source regions. IEEE Transact. Geosci. Remote Sens. 42, 557-569. https://doi.org/10.1109/TG RS.2004.824067

Kaufman, Y.J., Tanré, D., Remer, L.A., Vermote, E.F., Chu, A., Holben, B.N. (1997a). Operational remote sensing of tropospheric aerosol over land from EOS moderate resolution imaging spectroradiometer. J. Geophys. Res. 102, 51-17. https://doi.org/10.1029/96JD03988

Kaufman, Y.J., Wald, A.E., Remer, L.A., Gao, B.C., Li, R.R., Flynn, L. (1997b). The MODIS 2.1- $\mu$ m 
channel-correlation with visible reflectance for use in remote sensing of aerosol. IEEE Transact. Geosci. Remote Sens. 35, 1286-1298. https://doi.org/10.1109/36.628795

Kaufman, Y.J., Sendra, C. (1988). Algorithm for automatic atmospheric corrections to visible and near-IR satellite imagery. Int. J. Remote Sens. 9, 1357-1381. https://doi.org/10.1080/0143116 8808954942

King, M.D., Kaufman, Y.J., Tanré, D. (1999). Remote sensing of tropospheric aerosols from space: Past, present and future. Bull. Am. Meteorol. Soc. 80, 2229-2260. https://doi.org/10.1175/152 0-0477(1999)080<2229: RSOTAF>2.0.CO

Levy, R.C., Remer, L.A., Mattoo, S., Vermote, E.F., Kaufman, Y.J. (2007). Second-generation operational algorithm: Retrieval of aerosol properties over land from inversion of moderate resolution imaging spectroradiometer spectral reflectance. J. Geophys. Res. 112, D13211. https://doi.org/10.1029/2006JD007811

Levy, R.C., Mattoo, S., Munchak, L.A., Remer, L.A., Sayer, A.M., Patadia, F., Hsu, N.C. (2013). The collection 6 MODIS aerosol products over land and ocean. Atmos. Meas. Tech. 6, 2989-3034. https://doi.org/10.5194/amt-6-2989-2013

Li, X., Xia, X., Wang, S., Mao, J., Liu, Y. (2012). Validation of MODIS and Deep Blue aerosol optical depth retrievals in an arid/semi-arid region of northwest China. Particuology 10, 132-139. https://doi.org/10.1016/j.partic.2011.08.002

Sayer, A.M., Hsu, N.C., Bettenhausen, C., Jeong, M.J. (2013). Validation and uncertainty estimates for MODIS Collection 6 "Deep Blue" aerosol data. J. Geophys. Res. 118, 7864-7872. https://doi.org/10.1002/jgrd.50600

Sun, L., Wei, J., Jia, C., Yang, Y., Zhou, X., Gan, P., Liu, F., Jia, S., Li, R. (2016b). A high-resolution global dataset of aerosol optical depth over land from MODIS data. In 2016 IEEE International Geoscience and Remote Sensing Symposium (IGARSS), 10-15 July, 2016, Beijing, China, IEEE. https://doi.org/10.1109/IGARSS.2016.7730497

Sun, L., Yu, H., Fu, Q., Wang, J., Tian, X., Mi, X. (2016a). Aerosol optical depth retrieval and atmospheric correction of GF-1 PMS supported by surface reflectance products. J. Remote Sens. 20, 216-228. https://doi.org/10.11834/jrs.20165052

Tanre, D., Holben, B.N., Kaufman, Y.J. (1992). Atmospheric correction algorithm for NOAA-AVHRR products - Theory and application. IEEE Transact. Geosci. Remote Sens. 30, 231-248. https://doi.org/10.1109/36.134074

Wang, H., Wang, Y., Yuan, Q. (2021). Validation and spatiotemporal analysis of MODIS multi-angle atmospheric calibration aerosol products in China from 2008 to 2016. Remote Sens. Technol. Appl. 36, 217-228. https://doi.org/10.11873/j.issn.1004-0323.2021.1.0217 (in Chinese).

Wang, Q., Sun, L., Wei, J., Yang, Y., Li, R., Liu, Q., Chen, L. (2017). Validation and accuracy analysis of global MODIS aerosol products over land. Atmosphere 8, 155. https://doi.org/10.3390/atm os8080155

Wang, Q., Bi, Y.M., Liu, C., Yang, Z.D. (2020). Technical requirements and progress of airborne active remote sensing aerosol cloud parameters. Telem. Remote Control 41, 1-11. https://doi.org/10.13435/j.cnki.ttc.003099 (in Chinese).

Wang, X., Xin, J., Wang, L., Wang, Y. (2012). Aerosol optical depth observed by Chinese sun hazemeter network and comparison with MODIS products in three typical cities in China. Clim. Environ. Res. 17, 37-45. https://doi.org/10.3878/j.issn.1006-9585.2011.10034 (in Chinese).

Wei, J. (2017). Retrieval of global high resolution aerosol optical depth using remote sensing. Shandong University of Science and Technology, China.

Wei, J., Li, Z., Peng, Y., Sun, L. (2019). MODIS Collection 6.1 aerosol optical depth products over land and ocean: Validation and comparison. Atmos. Environ. 201, 428-440. https://doi.org/10. 1016/j.atmosenv.2018.12.004

Wei, J., Sun, L. (2018). An improved high-spatial-resolution aerosol retrieval algorithm for MODIS images over land. J. Geophys. Res. 123, 12291-12307. https://doi.org/10.1029/2017JD027795

Yang, Y.K., Sun, L., Wei, J. (2016). MODIS C5, C6 aerosol product validation and regional adaptability evaluation. J. Shandong Univ. Sci. Technol. 35, 17-27. https://doi.org/10.16452/j.c nki.sdkjzk.2016.05.002 (in Chinese).

Zhou, C.Y., Liu, Q.H., Tang, Y., Wang, K., Sun, L., He, Y.X. (2009). Comparative analysis of MODIS aerosol C004 and C005 products and evaluation of their applicability in northern China. J. Remote Sens. 13, 854-872. https://doi.org/10.3321/j.issn:1007-4619.2009.05.008 (in Chinese). 